\title{
Improving Detection of Multiple Sclerosis Lesions in the Posterior Fossa Using an Optimized 3D-FLAIR Sequence at 3T
}

\author{
(D) A. Lecler, (DI. El Sanharawi, (D). El Methni, (DO. Gout, (DP. Koskas, and (D). Savatovsky
}

\begin{abstract}
BACKGROUND AND PURPOSE: There is no consensus regarding the best MR imaging sequence for detecting MS lesions. The aim of our study was to assess the diagnostic value of optimized 3D-FLAIR in the detection of infratentorial MS lesions compared with an axial T2-weighted imaging, a 3D-FLAIR with factory settings, and a 3D double inversion recovery sequence.
\end{abstract}

MATERIALS AND METHODS: In this prospective study, 27 patients with confirmed MS were included. Two radiologists blinded to clinical data independently read the following sequences: axial T2WI, 3D double inversion recovery, standard 3D-FLAIR with factory settings, and optimized 3D-FLAIR. The main judgment criterion was the overall number of high-signal-intensity lesions in the posterior fossa; secondary objectives were the assessment of the reading confidence and the measurement of the contrast. A nonparametric Wilcoxon test was used to compare the MR images.

RESULTS: Twenty-two patients had at least 1 lesion in the posterior fossa. The optimized FLAIR sequence detected significantly more posterior fossa lesions than any other sequence: 7.5 versus $5.8,4.8$, and 4.1 ( $P$ values of .04, .03, and .03) with the T2WI, the double inversion recovery, and the standard FLAIR, respectively. The reading confidence index was significantly higher with the optimized FLAIR, and the contrast was significantly higher with the optimized FLAIR than with the standard FLAIR and the double inversion recovery.

CONCLUSIONS: An optimized 3D-FLAIR sequence improved posterior fossa lesion detection in patients with MS.

ABBREVIATIONS: CNR = contrast-to-noise ratio; DIR = double inversion recovery; FLAIRED = FLAIR-enhanced detection; PSIR = phase-sensitive inversion recovery; $\mathrm{SI}=$ signal intensity

M ultiple sclerosis is the most frequent chronic inflammatory disease of the central nervous system. ${ }^{1-4}$ MR imaging has been shown to be the most efficient technique to image $\mathrm{MS}^{5}$ and has been included in the diagnostic work-up of the disease since $2001 .^{6,7}$ The diagnosis of MS relies on proof of disease dissemination in space and time and exclusion of other disorders that can mimic MS. MR imaging is also the technique of choice in disease monitoring; in patients with clinically isolated syndromes sugges-

Received April 8, 2019; accepted after revision May 14

From the Departments of Neuroradiology (A.L., I.E.S., P.K., J.S.) and Neurology (O.G.), Fondation Ophtalmologique Adolphe de Rothschild, Paris, France; and Department of Biostatistics (J.E.M.), MAP5 Laboratory, Unité Mixte de Recherche Centre National de la Recherche Scientifique 8145, Paris Descartes University, Sorbonne Paris Cité, Paris, France.

Augustin Lecler and Imane El Sanharawi contributed equally to the work and are co-first authors.

Please address correspondence to Augustin Lecler, MD, PhD, Department of Neuroradiology, Fondation Ophtalmologique Adolphe de Rothschild, 25 rue Manin, 75019 Paris, France; e-mail: alecler@for.paris; @RothNeuroRad

Indicates article with supplemental on-line photo.

http://dx.doi.org/10.3174/ajnr.A6107 tive of MS, it has substantial prognostic value in the prediction of the conversion to definite MS. ${ }^{8,9}$

MR imaging is performed in suspected or confirmed MS as a multisequence protocol, including minimally T2-weighted imaging and FLAIR sequences. ${ }^{10-14}$ The key sequence to detect brain demyelinating lesions is the $3 \mathrm{D}$-FLAIR sequence, which offers better sensitivity than the 2D-FLAIR sequence and T2WI. ${ }^{15,16}$ 3D-FLAIR can achieve homogeneous CSF suppression, is minimally affected by artifacts, ${ }^{15,17,18}$ and provides a better resolution due to smaller slice thickness. ${ }^{19}$ It provides a higher contrast-tonoise ratio and allows multiplanar reconstructions. Its use is supported by international guidelines. ${ }^{10,12}$ However, FLAIR sequences were historically reported to be less sensitive than T2WI in the posterior fossa, ${ }^{20}$ and conflicting results have been reported in the literature so far. ${ }^{15,18,19,21,22}$

Many sequences have been tested to improve the detection of demyelinating encephalic lesions in patients with MS, such as magnetization transfer, ${ }^{23}$ MPRAGE ${ }^{24}$ double inversion recovery (DIR), ${ }^{19,25-27}$ the phase-sensitive inversion recovery (PSIR) sequence, ${ }^{28,29}$ or $3 \mathrm{D}$ FLAIR-enhanced detection 
Table 1: MRI sequence parameters

\begin{tabular}{lcccc}
\hline & TSE T2WI & DIR & FLAIR 4800 & FLAIR 8000 \\
\hline TR (ms) & 4545 & 5500 & 4800 & 8000 \\
TE (ms) & 80 & 277 & 245 & 328 \\
TI (ms) & NA & 2550 & 1650 & 2400 \\
FOV (mm) & $230 \times 230$ & $250 \times 250$ & $240 \times 240$ & $240 \times 240$ \\
Acquisition matrix (mm) & $384 \times 299$ & $200 \times 200$ & $240 \times 243$ & $240 \times 243$ \\
Reconstruction matrix (mm) & $512 \times 512$ & $256 \times 256$ & $256 \times 256$ & $256 \times 256$ \\
Acquisition plane & Axial & Sagittal & Sagittal & Sagittal \\
Slice thickness (mm) & 3 & 1.4 & 1 & 1 \\
Gap (mm) & 0 & 0 & 0 & 0 \\
No. of slices & 47 & 277 & 365 & 365 \\
Flip angle & $90^{\circ}$ & $90^{\circ}$ & $90^{\circ}$ & $90^{\circ}$ \\
Echo-train & 15 & 175 & 130 & 180 \\
NEX & 1 & 1 & 1 & 1 \\
Fat-suppression & None & SPIR & SPIR & SPIR \\
Bandwidth/pixel (Hz) & 202 & 1179 & 938 & 938 \\
Scan time duration & 2 min 34 sec & 3 min 51 sec & 3 min 41 sec & 4 min 24 sec \\
Mode & $2 D$ & $3 D$ & $3 D$ & $3 D$ \\
\hline
\end{tabular}

Note:-SPIR indicates spectral presaturation with inversion recovery; NA, not applicable; TSE, turbo spin echo.

and an optimized 3D-FLAIR, which provided the best subjective contrast on a preliminary testing on 3 patients with MS not included in our study, as planned in the study design. We optimized this 3D-FLAIR by lengthening its TR to $8000 \mathrm{~ms}$, modifying its TI value to allow satisfactory fluid suppression, increasing its turbo factor to partially compensate for the increased acquisition time induced by the higher TR, and, to maintain clinically acceptable examination duration, also setting its effective TE to be as short as possible.

The order of the sequences was randomized between participants to overcome a possible effect of contrast agent administration on lesion detection. An intravenous contrast injection of a single

(FLAIRED) ${ }^{18}$ However, the posterior fossa remains a challenging area for MR imaging, and the quality of images and the sharpness of lesion visualization are not always optimal. ${ }^{20}$ Yet, detecting posterior fossa lesions is essential for a diagnosis of MS and is 1 of the 4 areas of the CNS that must be explored according to the McDonald criteria. ${ }^{7}$ Lesion burden was reported to be highly correlated with long-term disability and lower sensory Functional System Scores. ${ }^{30,31}$

The aim of our study was to evaluate the diagnostic value of an optimized 3D-FLAIR sequence at 3T to detect infratentorial MS lesions.

\section{MATERIALS AND METHODS Study Design}

We conducted a prospective study (NCT 03108573) in a tertiary referral center (Fondation Ophtalmologique Adolphe de Rothschild, Paris, France) specializing in neurologic diseases. This study was approved by a national independent research ethics board and adhered to the tenets of the Declaration of Helsinki (institutional review board 2012-A00993-40). Signed informed consent was obtained from all subjects. This study follows the Standards for Reporting Diagnostic Accuracy Studies guidelines. $^{32}$

Twenty-seven patients were included. Inclusion criteria were older than 18 years of age with a confirmed diagnosis of MS as defined by the 2017 McDonald criteria. ${ }^{7}$ Patients with any MR imaging contraindication were not included. Exclusion criteria were insufficient quality for interpretation of any of the evaluated sequences and absence of our optimized 3DFLAIR sequence with a TR of $8000 \mathrm{~ms}$. Selection of patients is shown in the On-line Figure.

\section{MR Imaging Protocol}

All MRIs were performed on the same 3T Ingenia device (Philips Healthcare, Best, the Netherlands) with a 16-channel neurovascular coil. Each patient underwent the following standard MS protocol: axial turbo spin echo (TSE) T2WI and a standard 3DFLAIR with factory settings as provided by the vendor. This protocol was completed by 2 supplementary sequences: a 3D-DIR dose of gadobutrol (Gadovist; Bayer Schering Pharma, Berlin, Germany) was performed for all patients at least 5 minutes before the MR imaging acquisition, as recommended by international guidelines. ${ }^{12}$ Technical specifications are described in Table 1.

\section{Image and Data Analysis}

Two radiologists, blinded to patient identification, medical history, and lab results, independently read, in random order, the MR images. The first one was a senior neuroradiologist with 20 years of experience (P.K.), and the second was a junior radiologist with no experience in neurologic imaging (I.E.S.). Each of the 4 sequences from 1 patient was read separately and independently from the other sequences. Readers were not allowed to read $>1$ sequence of the same patient during the same reading session. A second reading was performed 3 weeks later. Discrepancies were resolved by a third radiologist specialized in neuroradiology with 7 years of experience (A.L.). All data were read on a dedicated workstation with the OsiriX imaging software (http://www. osirix-viewer.com).

The primary judgment criterion was the overall number of highsignal lesions detected in the posterior fossa. We also assessed the following MR imaging characteristics: the location of the lesions (either in the cerebellum or the brain stem); the length of the largest lesion in each location; and the confidence in the detection of the lesions, estimated as follows: 1 corresponding to a low confidence; 2 , to a moderate-to-high confidence; and 3 , to a very high confidence.

\section{Signal and Contrast Properties}

We measured the SNR using the National Electrical Manufacturers Association method, ${ }^{33}$ which needed 2 images acquired within 5 minutes of each other with the exact same imaging parameters. Those 2 images were then subtracted to produce a third one. On each subtracted sequence for each patient, we placed 2 ROIs in the white matter of the right and left cerebellar hemispheres to measure their signal intensity (SI) and obtain the mean white matter $\mathrm{SI}\left(\mathrm{SI}_{\mathrm{WM}}\right)$. We took the SD of the value of the ROIs and extracted the average SD of the noise. For the contrast-to-noise ratio (CNR) of the lesions, we manually placed an ROI in each sequence for 


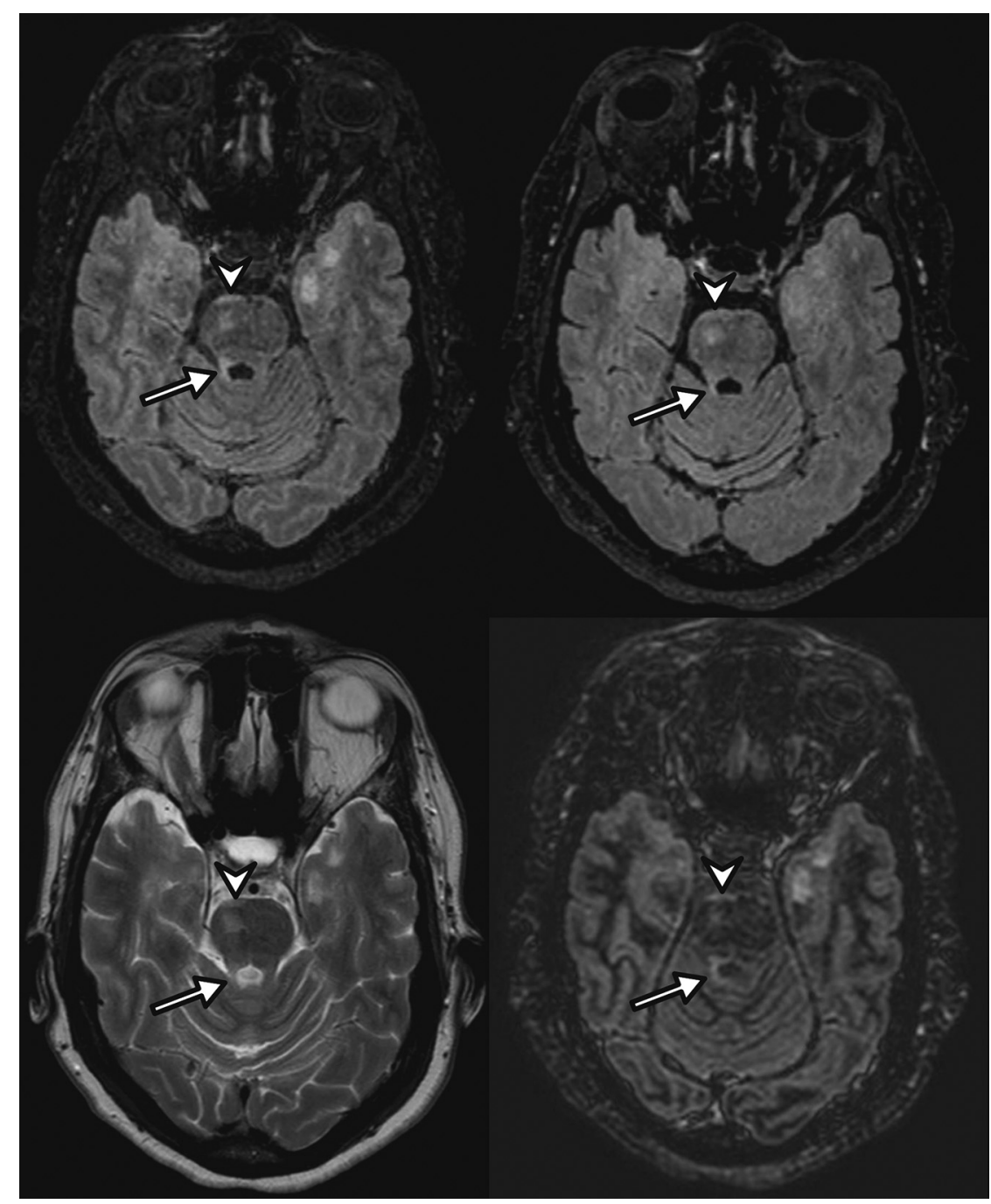

FIG 1. A 35-year-old woman with relapsing-remitting multiple sclerosis. 3D-optimized FLAIR (upper left), 3D standard FLAIR (upper right), axial T2WI (lower left), and 3D-DIR (lower right) MR imaging show a round lesion of the pons well-visualized on all sequences (arrowhead) and a lesion of the right superior cerebellar peduncle (arrow) more conspicuous on the optimized FLAIR sequence.

each patient in 2 nonenhancing lesions of significant size to measure their SI and obtain the mean lesion SI $\left(\mathrm{SI}_{\text {lesion }}\right)$. Those lesions had to be visible on all sequences and were the same on each sequence. Then, we calculated the SNR using the formula $S N R=\frac{S I_{\mathrm{WM}} \times \sqrt{2}}{S D}$ and the CNR as follows: $C N R=$ $\frac{S I_{\text {lesion }}-S I_{\mathrm{WM}}}{S D}$.

\section{Statistical Analysis}

Analyses were conducted by a statistical analyst (J.E.M.) with 10 years of experience using R software, Version 3.3.2. ${ }^{34}$ Categoric data were reported as number (percentage) as appropriate. Continuous data were reported as median with interquartile ranges or means $\pm S D$ as appropriate. The nonparametric Wilcoxon test was used to compare the different MR images, using the Streitberg-Röhmel algorithm in case of a tie. Because multiple tests were performed, the Bonferroni adjustment was used with an $\alpha$ level adjusted to a significance level of .005 . $P$ values $<.05$ were considered significant. The Cohen $\kappa$ statistic was used to assess inter- and intraobserver agreement for the MR imaging reading and was interpreted as follows: $0-0.20$, poor agreement; $0.21-0.40$, fair agreement; $0.41-0.60$, moderate agreement; $0.61-0.80$, good agreement; $0.81-1$, almost perfect agreement. ${ }^{35}$ The sample size was calculated on the basis of a minimum expected mean difference of $10 \%$ in detecting MS lesions between the optimized FLAIR and the T2WI and a common SD of 0.15 . The statistical power was set at 0.9 , and the significance criterion was set to .05 , with a 2 -tailed analysis. ${ }^{36}$ Twenty-one patients would have been necessary for this statistical analysis. A final objective of 27 patients was set to anticipate secondary exclusions and unusable data.

\section{RESULTS}

\section{Patient Characteristics}

Twenty-seven patients with a confirmed diagnosis of MS (14 men and 13 women; mean age, $37 \pm 10.7$ years) were included. The mean Expanded Disability Status score was $3.2 \pm 2.1$. The mean disease duration was $13.6 \pm 9.2$ years. The MS phenotype composition was as follows: 21 (78\%) relapsing-remitting; 4 (15\%) secondary-progressive; and 2 (7\%) primitive-progressive.

\section{Infratentorial Lesions}

Of all 27 patients, 22 had at least 1 lesion in the posterior fossa detected with 1 of the 4 sequences, with a mean number of $5.6 \pm 7.5$ lesions per patient. There were $55,57,49$, and 85 lesions in the cerebellum detected on T2WI, DIR, standard FLAIR, and optimized FLAIR, respectively. There were 102, 69, 63, and 108 lesions in the brain stem detected on T2WI, DIR, standard FLAIR, and optimized FLAIR, respectively. The largest lesion had a median length of $7.7 \mathrm{~mm}$ (interquartile range, 4.8 $11.9 \mathrm{~mm}$ ) and $9.2 \mathrm{~mm}$ (interquartile range, $5.9-12.0 \mathrm{~mm}$ ) in the cerebellum and the brain stem, respectively.

Optimized FLAIR detected significantly more posterior fossa lesions than with T2WI, DIR, or standard FLAIR: $7.5 \pm 9$ versus $5.8 \pm 8.6(P=.04), 4.8 \pm 7(P=.03)$, and $4.1 \pm 4.6(P=.03)$, respectively, as shown on Figs 1 and 2. Subgroup comparisons are detailed in Table 2. Eight, 9, and 3 patients had no posterior fossa lesion detected with T2WI, DIR, and standard FLAIR, respectively, whereas they had at least 1 lesion detected with optimized FLAIR. Detailed intersequence comparisons are shown in Table 3.

The median values of the SNR and CNR were significantly higher with T2WI. The optimized FLAIR showed a higher CNR than DIR and standard FLAIR (Table 2).

The mean reading confidence index was significantly higher with 
the optimized FLAIR sequence than with any other sequences: 2.8 versus $1.9\left(P=5.10^{-6}\right), 2.3\left(P=5.10^{-3}\right)$, and $2.0\left(P=3.10^{-6}\right)$ for T2WI, DIR, and the standard FLAIR sequences, respectively.

\section{Inter- and Intraobserver Reproducibility}

Interreader agreement was moderate for the overall number of lesions and for the cerebellum location and good for the brain stem location ( $\kappa$ values $=0.58,0.49$, and 0.67 , respectively). Intraobserver agreement between the 2 readings was excellent for

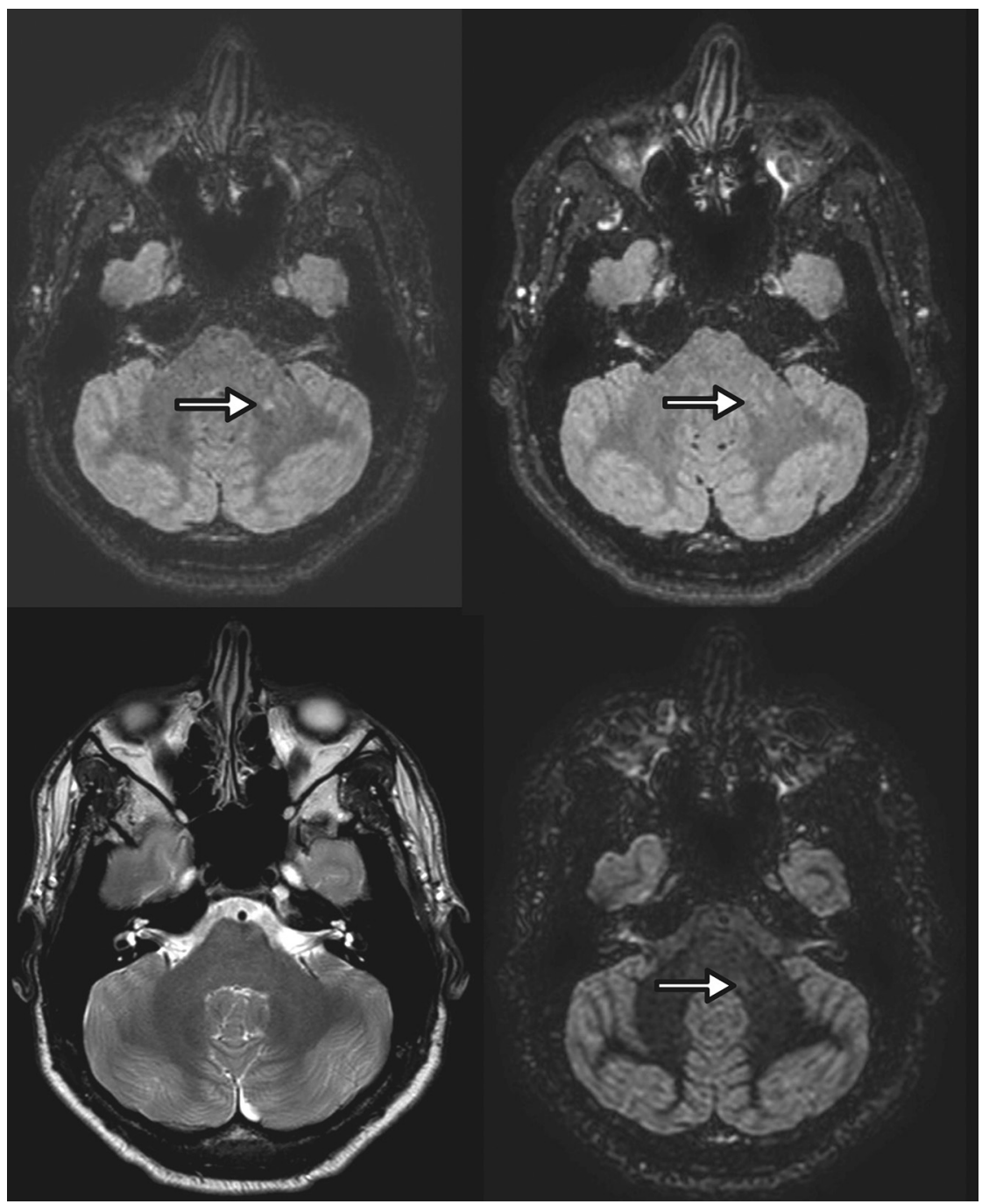

FIG 2. A 29-year-old woman with relapsing-remitting multiple sclerosis. 3D-optimized FLAIR (upper left), standard 3D-FLAIR (upper right), and 3D-DIR (lower right) MR imaging show a left middle cerebellar peduncle lesion not visible on the axial T2WI sequence (lower left). Note that the lesion is more conspicuous on the optimized FLAIR sequence. the first reader and fair for the second reader $(\kappa$ values $=0.90$ and 0.33 , respectively).

\section{DISCUSSION}

Our study showed that an optimized 3D-FLAIR sequence significantly improved the overall posterior fossa lesion detection in patients with MS, with a significantly higher reader-reported confidence, supporting a significantly higher number of detected lesions than would have normally been found with a T2WI, standard 3D-FLAIR with factory settings, or DIR sequence.

Many studies have tried to find the best MR image to accurately detect demyelinating encephalic lesions in patients with MS, such as T2WI, ${ }^{5}$ protondensity-weighted, ${ }^{15}$ 2D-FLAIR, ${ }^{19,37,38}$ magnetization transfer, ${ }^{23}$ MPRAGE, ${ }^{24}$ 3D-FLAIR, ${ }^{15,16,18,19,22}$ DIR, ${ }^{19,25-27}$ or PSIR. ${ }^{28,29}$ There have also been studies trying to improve the detection rate by combining sequences, such as the PT2 ${ }^{39}$ or FLAIR, ${ }^{40}$ or by subtraction. ${ }^{19}$ However, we found only 1 study that specifically dealt with 3T 3D-FLAIR sequence optimization for identifying MS lesions by modifying the TE. In this study, the authors developed a 3D-FLAIRED with an iterative approach based on theoretic considerations. They obtained and analyzed a 3D spin-echo FLAIR with a longer contrast-equivalent TE than the effective TE of a 3D-FLAIR (600 versus $450 \mathrm{~ms}$ ) without modifying the TR values held constant at $7000 \mathrm{~ms}$. They showed that lesion recognition was improved in both supratentorial and infratentorial regions and that detection of cortical lesions increased with the 3DFLAIRED. ${ }^{18}$ Our study is the first to evaluate the value of a FLAIR sequence with a long TR versus conventional 3DFLAIR, axial T2WI, and DIR in the detection rate of infratentorial MS lesions specifically.

Our results are in agreement with those obtained by previously published studies, in which the FLAIR sequence

Table 2: Description of the posterior fossa lesions and comparison of mean values of SNR and CNR

\begin{tabular}{|c|c|c|c|c|c|c|c|c|c|c|}
\hline & $\mathrm{T} 2$ & DIR & $\begin{array}{l}\text { Standard } \\
\text { FLAIR }\end{array}$ & $\begin{array}{l}\text { Optimized } \\
\text { FLAIR }\end{array}$ & T2/DIR ${ }^{a}$ & $\begin{array}{l}\text { T2/Standard } \\
\text { FLAIR }^{\mathrm{a}}\end{array}$ & $\begin{array}{l}\text { T2/Optimized } \\
\text { FLAIR }^{\mathrm{a}}\end{array}$ & $\begin{array}{c}\text { Standard } \\
\text { FLAIR/DIR }^{\mathrm{a}}\end{array}$ & $\begin{array}{l}\text { Optimized } \\
\text { FLAIR/DIR }^{\mathrm{a}}\end{array}$ & $\begin{array}{c}\text { Optimized } \\
\text { FLAIR/Standard } \\
\text { FLAIR }^{\mathrm{a}}\end{array}$ \\
\hline \multicolumn{11}{|c|}{ Mean (SD) No. of lesions } \\
\hline All locations & $5.8(8.6)$ & $4.8(7.0)$ & $4.1(4.6)$ & $7.5(9.0)$ & 6 & .4 & $.04^{\mathrm{b}}$ & .3 & $.03^{\mathrm{b}}$ & $.03^{\mathrm{b}}$ \\
\hline Cerebellum & $2.0(4.5)$ & $2.2(3.6)$ & $1.8(2.1)$ & $3.5(4.8)$ & .1 & $.05^{\mathrm{b}}$ & $7.10^{-4 b}$ & .4 & $.01^{\mathrm{b}}$ & $.02^{\mathrm{b}}$ \\
\hline Brain stem & $3.8(4.8)$ & $2.7(3.9)$ & $2.3(2.8)$ & $4.0(4.9)$ & .2 & .3 & .3 & .4 & .2 & .1 \\
\hline Mean SNR (SD) & 112 (17.1) & $16(2.3)$ & $34(4.4)$ & $29(4.2)$ & $5.10^{-5 b}$ & $8.10^{-5 b}$ & $7.10^{-5 b}$ & $2.10^{-3 b}$ & $3.10^{-3 b}$ & .1 \\
\hline Mean CNR (SD) & 45 (7.9) & $9(1.7)$ & $11(1.9)$ & $14(2.2)$ & $4.10^{-4 b}$ & $4.10^{-4 b}$ & $6.10^{-4 b}$ & .1 & $.01^{\mathrm{b}}$ & $.03^{b}$ \\
\hline
\end{tabular}

a $P$ value after comparison of the 2 groups with the nonparametric Wilcoxon test.

b Significant differences after appropriate statistical correction. 
Table 3: Number of patients with at least 1 lesion detected by 1 sequence only

\begin{tabular}{lcccc}
\hline & \multicolumn{4}{c}{$\begin{array}{c}\text { Patients with at Least 1 Lesion } \\
\text { Detected }\end{array}$} \\
\cline { 2 - 5 } & T2WI & DIR & $\begin{array}{c}\text { Standard } \\
\text { FLAIR }\end{array}$ & $\begin{array}{c}\text { Optimized } \\
\text { FLAIR }\end{array}$ \\
\hline $\begin{array}{l}\text { Patients with no } \\
\text { lesion detected }\end{array}$ & & & & \\
T2WI & 2 & 1 & 5 & 8 \\
DIR & 1 & 1 & 6 & 9 \\
Standard FLAIR & 0 & 0 & 0 & 3 \\
$\quad$ Optimized FLAIR & & & \\
\hline
\end{tabular}

was reported to depict more lesions than a conventional T2WI sequence. ${ }^{15,16,18,19,22,25,26,37}$ We did not find significant differences in the detection rate of infratentorial MS lesions among DIR, axial T2WI, and standard FLAIR, a finding concordant with the literature. ${ }^{19,25}$ However, we observed a significantly higher detection rate of MS lesions with the optimized FLAIR sequence than with the DIR sequence, as opposed to Wattjes et $\mathrm{al},{ }^{26}$ who compared a DIR sequence with a FLAIR $_{12,000}$ sequence. Moreover, optimized FLAIR detected at least 1 posterior fossa lesion in 8,9 , and 3 patients with no lesions detected on T2WI, DIR, and standard FLAIR, respectively, which is clinically very important because the posterior fossa is 1 of the 4 main locations taken into account for diagnosing MS. ${ }^{7}$ This might support earlier diagnosis and improve management of patients with MS. It might also be relevant for follow-up to avoid misclassifying patients as having no evidence of disease activity, which is increasingly considered the treatment goal. ${ }^{41}$

The posterior fossa is a challenging area for MR imaging. Image quality and sharpness of lesions are not always optimal, and the 2D-FLAIR sequence was historically reported as less sensitive than T2WI in this location, ${ }^{20}$ mostly because of artifacts due to CSF and blood inflow or insufficient T2WI. ${ }^{37}$ The use of 3DFLAIR sequences overcomes these issues because they can achieve homogeneous CSF suppression, are minimally affected by artifacts, ${ }^{15,17,18}$ and provide a better resolution due to smaller slice thickness. ${ }^{19}$ The better performance of the optimized FLAIR sequence over the standard FLAIR may be explained by the intrinsic characteristics of the FLAIR sequence. The main parameters affecting the contrast of the FLAIR sequence are the TR, TE, TI, and flip angles. The TR seems to be the most relevant parameter to study because it is the main determinant of the intensity of the T2 signal. It is related to the T2 effect, and the augmentation of the effective TR attenuates the normal white matter signal, making lesions more easily depictable. ${ }^{18,42}$ We subjectively chose a TR of $8000 \mathrm{~ms}$ for our optimized 3D-FLAIR sequence because it provided the best subjective contrast on preliminary testing in our center. Most interesting, this value is very close to the TR of 7000 ms of the optimized FLAIRED sequence, which was considered as the most sensitive in the detection of MS lesions in both supraand infratentorial regions ${ }^{18}$ and is close to the TR values of $8000-$ $10,000 \mathrm{~ms}$ proposed to reach near-optimum contrast and high multisection efficiency as in a previous 2D-FLAIR optimization study at $1.5 \mathrm{~T}^{42}$

A longer TR significantly increased the CNR in our series, allowing higher confidence in discriminating demyelinating lesions from normal-appearing white matter. We compared the optimized FLAIR with the standard 3D-FLAIR with factory settings provided by most of the vendors at the time, with a TR of 4800 ms. This standard 3D-FLAIR is tested and normed on healthy volunteers and is not optimized to depict pathologic abnormalities of signal, even though it offers a good CSF suppression.

The DIR sequence, after attenuating the signal of both the white matter and the CSF, ${ }^{43}$ was reported to allow a better delineation between white and gray matter and seems to be a sequence of choice for the detection of supratentorial lesions, ${ }^{19,25}$ especially the cortical ones. However, we did not note its superior detection in our study, probably due to increased noise at the CSF interfaces.

The most recent guidelines of the Consortium of Multiple Sclerosis Centers group ${ }^{12}$ include a whole-brain 3D-T2-FLAIR and a 3D-T2WI, and the Magnetic Resonance Imaging in MS group $^{10}$ recommended a standardized protocol, including axial T2WI and proton-density or T2-FLAIR sequences, and sagittal $2 \mathrm{D}$ or isotropic $3 \mathrm{D}-\mathrm{T} 2-\mathrm{FLAIR}$ sequences. Our results suggest that the need for performing both a 3D-FLAIR sequence and a T2WI sequence for imaging of the posterior fossa in patients with MS might be questioned because no lesion seen on T2WI was missed on the optimized 3D-FLAIR either in our study or in the literature. ${ }^{15}$ We might advantageously reduce the MR imaging examination time by removing the T2WI sequence from our protocols.

Both the Consortium of Multiple Sclerosis Centers and the Magnetic Resonance Imaging in MS groups also recommend minimal technical specifications such as the maximum slice thickness, spatial resolution, voxel size, and duration time of the protocol. We believe that guidelines should also include recommendations on key parameters such as the TR. A TR of $8000 \mathrm{~ms}$ seems to be a good compromise for the FLAIR sequence, with reasonable acquisition time, good SNR and CNR, and a high confidence index, meaning an easier and more comfortable reading. The FLAIR sequence is widely available in all centers, and the modification of its parameters might be easily feasible on most clinical scanners. No extra time or specific software is needed for postprocessing, compared with other performant-sensitive sequences. ${ }^{39,40}$ It is simple and fits the clinical setting, and it may facilitate standardization of protocols among different centers worldwide.

An increase in the detection of posterior fossa lesions presents 2 major direct advantages for the patient: an earlier diagnosis and prognosis classification (especially for clinically or radiologically isolated syndromes $)^{44,45}$ and a better follow-up due to an earlier diagnosis of new lesions. Those 2 elements have a deep impact on both the therapeutic decision-making and the patient's treatment. ${ }^{46,47}$ The presence of posterior fossa lesions is related to more substantial disability because the cerebellum and the brain stem serve critical functions and are often clinically eloquent locations. ${ }^{30,48,49}$ In our institution, we now routinely perform the optimized FLAIR sequence for suspected MS and MS follow-up.

Our study has some limitations: First, the results of the study are somewhat limited by the relatively small number of patients, and the results may be unique to the scanner and coil hardware used because no other systems were tested. Multicenter studies, including larger sample sizes, are necessary to confirm the results 
and to also validate them for other MR imaging manufacturers. Second, we did not include other very effective sequences in depicting MS lesions, such as PSIR or other advanced techniques such as PT2, FLAIRED, or FLAIR. ${ }^{2}$ Our study was not designed for this because its aim was to compare an optimized 3D-FLAIR with the most commonly used sequences used in clinical practice-that is, T2WI, standard 3D-FLAIR, and 3D-DIR.

Third, we compared 3D-FLAIR and DIR sequences with a 2DT2WI and not a 3D-T2WI, which might have increased the detection rate of the T2WI. However, the specifications of the 2DT2WI we used are compliant with international guidelines, and 2D-T2WI remains widely used to detect posterior fossa lesions worldwide.

Fourth, even though the examinations were anonymized and read in a random order and independently, the sequences tested had characteristics that could make them recognizable to readers, which could have led to a recognition bias.

Finally, we did not correlate results to pathology specimens and therefore cannot be sure that all lesions detected were, in fact, MS lesions. This correlation would have implied postmortem histologic examinations, which were not available.

\section{CONCLUSIONS}

Our study showed that an optimized 3D-FLAIR sequence significantly improved the overall posterior fossa lesion detection in patients with MS, with a significantly higher reader-reported confidence, supporting a significantly higher number of detected lesions than would have normally been found with a T2WI, standard 3D-FLAIR with factory settings, or DIR sequence.

\section{ACKNOWLEDGMENTS}

Laura McMaster provided professional English language medical editing of this article.

Disclosures: Jonathan El Methni—UNRELATED: Employment: Université Paris Descartes. Julien Savatovsky - UNRELATED: Payment for Lectures Including Service on Speakers Bureaus: Medtronic, Philips Healthcare, Sanofi, Biogen, Comments: Lecture fees were less than $€ 1000$; Travel/Accommodations/Meeting Expenses Unrelated to Activities Listed: Bayer Healthcare.

\section{REFERENCES}

1. Geurts JJG, Calabrese M, Fisher E, et al. Measurement and clinical effect of grey matter pathology in multiple sclerosis. Lancet Neurol 2012;11:1082-92 CrossRef Medline

2. Klaver R, De Vries HE, Schenk GJ, et al. Grey matter damage in multiple sclerosis: a pathology perspective. Prion 2013;7:66-75 CrossRef Medline

3. Messina S, Patti F. Gray matters in multiple sclerosis: cognitive impairment and structural MRI. Mult Scler Int 2014;2014:609694 CrossRef Medline

4. Pirko I, Lucchinetti CF, Sriram S, et al. Gray matter involvement in multiple sclerosis. Neurology 2007;68:634-42 CrossRef Medline

5. Runge VM, Price AC, Kirshner HS, et al. Magnetic resonance imaging of multiple sclerosis: a study of pulse-technique efficacy. $A J R$ Am J Roentgenol 1984;143:1015-26 CrossRef Medline

6. McDonald WI, Compston A, Edan G, et al. Recommended diagnostic criteria for multiple sclerosis: guidelines from the International Panel on the Diagnosis of Multiple Sclerosis. Ann Neurol 2001;50: 121-27 CrossRef Medline

7. Thompson AJ, Banwell BL, Barkhof F, et al. Diagnosis of multiple sclerosis: 2017 revisions of the McDonald criteria. Lancet Neurol 2018;17:162-73 CrossRef Medline
8. Barkhof F, Filippi M, Miller DH, et al. Comparison of MRI criteria at first presentation to predict conversion to clinically definite multiple sclerosis. Brain J Neurol 1997;120(Pt 11):2059-69 CrossRef Medline

9. Tintoré M, Rovira A, Martínez MJ, et al. Isolated demyelinating syndromes: comparison of different MR imaging criteria to predict conversion to clinically definite multiple sclerosis. AJNR Am J Neuroradiol 2000;21:702-06 Medline

10. Rovira À, Wattjes MP, Tintoré M, et al; MAGNIMS study group. Evidence-based guidelines: MAGNIMS consensus guidelines on the use of MRI in multiple sclerosis-clinical implementation in the diagnostic process. Nat Rev Neurol 2015;11:471-82 CrossRef Medline

11. Cotton F, Kremer S, Hannoun S, et al; Imaging Working Group of the Observatoire Français de la Sclérose en Plaques. OFSEP, a nationwide cohort of people with multiple sclerosis: consensus minimal MRI protocol. J Neuroradiol 2015;42:133-40 CrossRef Medline

12. Traboulsee A, Simon JH, Stone L, et al. Revised Recommendations of the Consortium of MS Centers Task Force for a Standardized MRI Protocol and Clinical Guidelines for the Diagnosis and Follow-Up of Multiple Sclerosis. AJNR Am J Neuroradiol 2016;37:394-401 CrossRef Medline

13. Simon JH, Li D, Traboulsee A, et al. Standardized MR imaging protocol for multiple sclerosis: Consortium of MS Centers consensus guidelines. AJNR Am J Neuroradiol 2006;27:455-61 Medline

14. Filippi M, Falini A, Arnold DL, et al; White Matter Study Group. Magnetic resonance techniques for the in vivo assessment of multiple sclerosis pathology: consensus report of the white matter study group. J Magn Reson Imaging 2005;21:669-75 CrossRef Medline

15. Gramsch C, Nensa F, Kastrup O, et al. Diagnostic value of 3D fluid attenuated inversion recovery sequence in multiple sclerosis. Acta Radiol 1987 2015;56:622-27 Medline

16. Tan IL, van Schijndel RA, Pouwels PJW, et al. Serial isotropic threedimensional fast FLAIR imaging: using image registration and subtraction to reveal active multiple sclerosis lesions. $A J R$ Am J Roentgenol 2002;179:777-82 CrossRef Medline

17. Barkhof F, Pouwels PJ, Wattjes MP. The Holy Grail in diagnostic neuroradiology: 3T or 3D? Eur Radiol 2011;21:449-56 CrossRef Medline

18. Polak P, Magnano C, Zivadinov R, et al. 3D FLAIRED: 3D fluid attenuated inversion recovery for enhanced detection of lesions in multiple sclerosis. Magn Reson Med 2012;68:874-81 CrossRef Medline

19. Moraal B, Roosendaal SD, Pouwels PJW, et al. Multi-contrast, isotropic, single-slab 3D MR imaging in multiple sclerosis. Eur Radiol 2008;18:2311-20 CrossRef Medline

20. Gawne-Cain ML, O’Riordan JI, Thompson AJ, et al. Multiple sclerosis lesion detection in the brain: a comparison of fast fluid-attenuated inversion recovery and conventional $\mathrm{T} 2$-weighted dual spin echo. Neurology 1997;49:364-70 CrossRef Medline

21. Tan IL, Pouwels PJW, van Schijndel RA, et al. Isotropic 3D fast FLAIR imaging of the brain in multiple sclerosis patients: initial experience. Eur Radiol 2002;12:559-67 CrossRef Medline

22. Bink A, Schmitt M, Gaa J, et al. Detection of lesions in multiple sclerosis by 2D FLAIR and single-slab 3D FLAIR sequences at 3.0 T: initial results. Eur Radiol 2006;16:1104-10 CrossRef Medline

23. Iannucci G, Rovaris M, Giacomotti L, et al. Correlation of multiple sclerosis measures derived from T2-weighted, T1-weighted, magnetization transfer, and diffusion tensor MR imaging. AJNR Am J Neuroradiol 2001;22:1462-67 Medline

24. Nelson F, Poonawalla A, Hou P, et al. 3D MPRAGE improves classification of cortical lesions in multiple sclerosis. Mult Scler 2008;14: 1214-19 CrossRef Medline

25. Vural G, Keklikoğlu HD, Temel Ş, et al. Comparison of double inversion recovery and conventional magnetic resonance brain imaging in patients with multiple sclerosis and relations with disease disability. Neuroradiol J 2013;26:133-42 CrossRef Medline

AJNR Am J Neuroradiol 40:1170-76 Jul 2019 www.ajnr.org 
26. Wattjes MP, Lutterbey GG, Gieseke J, et al. Double inversion recovery brain imaging at 3T: diagnostic value in the detection of multiple sclerosis lesions. AJNR Am J Neuroradiol 2007;28:54-59 Medline

27. Geurts JJ, Pouwels PJ, Uitdehaag BM, et al. Intracortical lesions in multiple sclerosis: improved detection with 3D double inversion-recovery MR imaging. Radiology 2005;236:254-60 CrossRef Medline

28. Nelson F, Poonawalla AH, Hou P, et al. Improved identification of intracortical lesions in multiple sclerosis with phase-sensitive inversion recovery in combination with fast double inversion recovery MR imaging. AJNR Am J Neuroradiol 2007;28:1645-49 CrossRef Medline

29. Favaretto A, Poggiali D, Lazzarotto A, et al. The parallel analysis of phase sensitive inversion recovery (PSIR) and double inversion recovery (DIR) images significantly improves the detection of cortical lesions in multiple sclerosis (MS) since clinical onset. PLoS One 2015;10:e0127805 CrossRef Medline

30. Minneboo A, Barkhof F, Polman CH, et al. Infratentorial lesions predict long-term disability in patients with initial findings suggestive of multiple sclerosis. Arch Neurol 2004;61:217-21 CrossRef Medline

31. Quattrocchi CC, Cherubini A, Luccichenti G, et al. Infratentorial lesion volume correlates with sensory functional system in multiple sclerosis patients: a 3.0-Tesla MRI study. Radiol Med 2010;115: 115-24 CrossRef Medline

32. Bossuyt PM, Reitsma JB, Bruns DE, et al; STARD Group. STARD 2015: an updated list of essential items for reporting diagnostic accuracy studies. Radiology 2015;277:826-32 CrossRef Medline

33. Goerner FL, Clarke GD. Measuring signal-to-noise ratio in partially parallel imaging MRI. Med Phys 2011;38:5049-57 CrossRef Medline

34. The R Foundation. The R Project for Statistical Computing. https:// www.R-project.org. Accessed April 23, 2017

35. Landis JR, Koch GG. An application of hierarchical kappa-type statistics in the assessment of majority agreement among multiple observers. Biometrics 1977;33:363-74 CrossRef Medline

36. Eng J. Sample size estimation: how many individuals should be studied? Radiology 2003;227:309-13 CrossRef Medline

37. Okuda T, Korogi Y, Shigematsu Y, et al. Brain lesions: when should fluid-attenuated inversion-recovery sequences be used in MR evaluation? Radiology 1999;212:793-98 CrossRef Medline
38. Hashemi RH, Bradley WG Jr, Chen DY, et al. Suspected multiple sclerosis: MR imaging with a thin-section fast FLAIR pulse sequence. Radiology 1995;196:505-10 CrossRef Medline

39. Gaitán MI, Yañes P, Sati P, et al. Optimal detection of infratentorial lesions with a combined dual-echo MRI sequence: "PT2." Mult Scler 2016;22:1367-70 CrossRef Medline

40. Wiggermann V, Hernández-Torres E, Traboulsee A, et al. FLAIR2: a combination of FLAIR and T2 for improved MS lesion detection. AJNR Am J Neuroradiol 2016;37:259-65 CrossRef Medline

41. Bevan CJ, Cree BA. Disease activity free status: a new end point for a new era in multiple sclerosis clinical research? JAMA Neurol 2014; 71:269-70 CrossRef Medline

42. Rydberg JN, Riederer SJ, Rydberg CH, et al. Contrast optimization of fluid-attenuated inversion recovery (FLAIR) imaging. Magn Reson Med 1995;34:868-77 CrossRef Medline

43. Redpath TW, Smith FW. Technical note: use of a double inversion recovery pulse sequence to image selectively grey or white brain matter. Br J Radiol 1994;67:1258-63 CrossRef Medline

44. Wattjes MP, Harzheim M, Kuhl CK, et al. Does high-field MR imaging have an influence on the classification of patients with clinically isolated syndromes according to current diagnostic MR imaging criteria for multiple sclerosis? AJNR Am J Neuroradiol 2006;27: 1794-98 Medline

45. Filippi M, Rocca MA, Ciccarelli O, et al; MAGNIMS Study Group. MRI criteria for the diagnosis of multiple sclerosis: MAGNIMS consensus guidelines. Lancet Neurol 2016;15:292-303 CrossRef Medline

46. Noyes K, Weinstock-Guttman B. Impact of diagnosis and early treatment on the course of multiple sclerosis. Am J Manag Care 2013;19(17 Suppl):s321-31 Medline

47. Tur C, Thompson AJ. Early accurate diagnosis crucial in multiple sclerosis. Practitioner 2015;259:21-7, 2-3 Medline

48. Tintore M, Rovira A, Arrambide G, et al. Brainstem lesions in clinically isolated syndromes. Neurology 2010;75:1933-38 CrossRef Medline

49. Preziosa P, Rocca MA, Mesaros S, et al. Relationship between damage to the cerebellar peduncles and clinical disability in multiple sclerosis. Radiology 2014;271:822-30 CrossRef Medline 